\title{
Cycloparaphenylene-Norbornene Monomers Afford Access to Carbon Nanohoop-Based Polymers
}

\author{
Ruth L. Maust, Penghao Li, Lev N. Zakharov, Ramesh Jasti*
}

\begin{abstract}
Although impressive strides have been made toward achieving precise polymer architectures, the pursuit of monomers with diverse structures and functions remains a critical challenge for polymer design. Herein we disclose the first polymers constructed from cycloparaphenylenes (CPPS), a family of strained, pi-rich macrocycles. Poly-CPPs were prepared via ring-opening metathesis polymerization (ROMP) of benzonorbornene-embedded CPPs. The distinctive size-dependent properties of CPPS, including fluorescence and host-guest chemistry, are preserved in poly-CPPs, offering a means to capitalize on these properties in polymeric materials. Moreover, copolymerizing CPPs of two different diameters results in polymers with emergent photophysical and supramolecular properties not achievable with small molecule CPP units. This work sets the stage for CPP derivatives to serve as molecular building blocks for the next generation of functional polymers.
\end{abstract}

Major breakthroughs in polymer chemistry in recent decades have enabled the preparation of polymers with complex architectures, controlled dispersities, and high degrees of functionality. ${ }^{[1-4]}$ With this unprecedented control over polymer construction, there now exists a unique opportunity to investigate the role of new "functional groups" in polymers-that is, new monomer structures which will provide access to new polymer properties and applications. A particularly attractive category of structural unit that has emerged in the realm of polymers is macrocycles (Fig. 1a)..$^{[5-10]}$ Although rare, macrocycle-based polymers demonstrate distinct advantages over polymers made up of acyclic units. Properties intrinsic to each type of macrocyclic unit, such as intrinsic porosity and affinity for guest molecules, make macrocycle-based polymers extremely well-suited for relevant challenges such as ion sensing ${ }^{[5]}$ and pollutant sequestration. ${ }^{[7,8,10,11]}$ Cyclic side chains can also improve polymer solubility, ${ }^{[12]}$ an advantage for materials processing. In a recent addition to this area, Hawker and coworkers showed that macrocyclic alkyl side chains in polymers retain the hydrophobicity of their linear counterparts while increasing the amorphous character of the polymers. ${ }^{[9]}$ Macrocycles also contribute intriguing possibilities for altering polymer morphology and resultant properties through noncovalent interactions, such as in supramolecular polymers,

[*] R. L. Maust; P. Li; Prof. R. Jasti

Department of Chemistry and Biochemistry and Materials Science Institute; Knight Campus for Accelerating Scientific Impact, University of Oregon

Eugene, OR 97403 (USA)

E-mail: riasti@uoregon.edu

L. N. Zakharov

CAMCOR, University of Oregon

Eugene, OR 97403 (USA)

Supporting information for this article is given via a link at the end of the document.

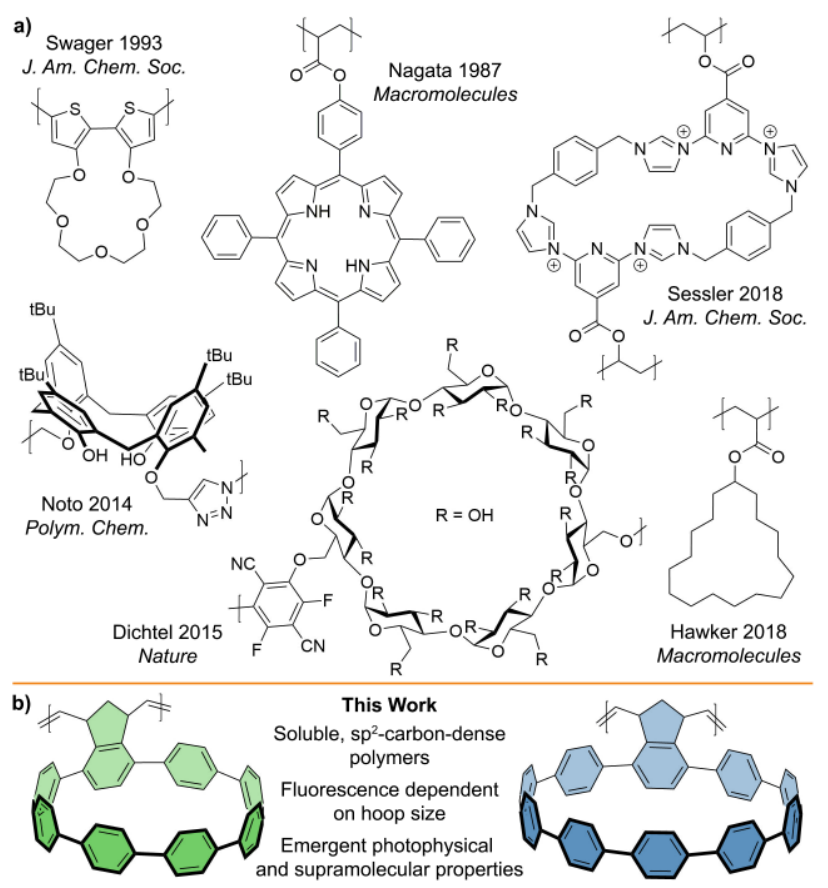

Figure 1. a) Macrocyclic units that have previously been incorporated into polymers include crown ethers, porphyrins, calixarenes, cyclodextrins, and cycloalkanes. b) Cycloparaphenylenes (CPPs) are the newest addition to the selection of macrocyclic building blocks for polymers.

dynamic polycatenanes, and slide-ring gels. ${ }^{[13-18]}$ While these examples highlight the many desirable features of macrocyclebased polymers, by far the most commonly employed macrocycles are cyclodextrins, leaving other classes of macrocycles relatively unexplored in polymeric materials. Given the value of cyclic structures in polymers, new types of macrocyclic side chains would be expected to result in new and useful properties.

One class of macrocycles that has never been incorporated into polymers previously is cycloparaphenylenes, or [ $n$ ]CPPs. Comprised of $n$ benzene rings linked end-to-end in the para position, these strained cyclic molecules can be thought of as the shortest fragments of armchair carbon nanotubes (CNTs). Unique photophysical properties arise from bending benzene into a hoop shape in this manner. For example, unlike in their linear counterparts, the HOMO-LUMO energy gap of CPPs decreases as the number of linked benzene rings decreases. This trend is also reflected in the red-shifting fluorescence emission as the hoop size decreases-[12]CPP has an emission maximum at $450 \mathrm{~nm}$ while [7]CPP emits at 587 $n m .{ }^{[19]}$ Small structural changes, such as incorporation of donoracceptor motifs ${ }^{[20]}$ or shifting the position of a bond, ${ }^{[21]}$ can also be used to tune the electronics and optical properties of these molecules. Beneficially, the distortion of the phenyl rings in 
CPPs disrupts pi-pi interactions among macrocycles, allowing CPPs with 12 or more unsubstituted phenyl rings to be readily soluble in common organic solvents. ${ }^{[20]}$ CPPs are also notable for their finely-tunable pore diameter and resultant supramolecular interactions, ${ }^{[22]}$ with the most prominent example being the strong binding of $\mathrm{C}_{60}$ in [10]CPP, marked by dramatic fluorescence quenching. ${ }^{[23,24]}$

In recent years, our group and others have devised synthetic methods to access highly functionalized CPPs to further tune their properties and investigate their potential applications. For instance, CPP derivatives are being developed as sensors, ${ }^{[25]}$ fluorescent probes, ${ }^{[26]}$ and blueprints for the bottom-up construction of discrete graphitic materials, predominantly carbon nanotubes. ${ }^{[27-29]}$ Encouraged by the broad applicability of macrocycle-containing polymers, we became interested in leveraging the unique properties of CPPs in polymeric materials. In addition, we expected that incorporating CPPs into polymers would provide extensive opportunities to alter the physical properties and processability of CPP-based nanomaterials without major synthetic modifications to the hoop backbone. Herein we report the first synthesis of CPPnorbornene (CPP-NB) monomers and CPP-based polymers obtained via ring-opening metathesis polymerization (ROMP) (Fig. 1b).

Given that the reactivity of CPPs can differ considerably from that of typical linear aromatic molecules, ${ }^{[30,31]}$ we first aimed to verify that CPPs could remain intact throughout polymerization. We selected ROMP as a polymerization method because of its high functional group tolerance and the ease of using this approach to obtain polymers of controlled molecular weight and complex sequences. ${ }^{[32,33]}$ We expected that by using ROMP we could avoid unwanted side reactions with the CPP backbone. Rather than appending a remote polymerizable group we opted for a direct linkage that would deliver macrocycledense polymers without overly influencing the polarity or electronic structure of the CPPs. Therefore, we devised a synthetic route to embed a ROMP-reactive benzonorbornene unit into the CPP backbone (Scheme 1). Taking into account the high strain inherent to CPPs, our customary synthetic strategy hinges on the use of curved intermediates containing cyclohexadienes as masked phenylenes. ${ }^{[34]}$ Incorporation of a benzonorbornene unit in the CPP backbone was accomplished using the same approach. Double nucleophilic addition of (4bromophenyl)lithium to norbornene-benzoquinone 1 followed by in situ methylation of the resulting alkoxides yielded dibromide 2. ${ }^{[35]}$ This curved intermediate served as a common coupling partner for forming multiple sizes of CPP-NB monomers in a modular manner. Coupling partner $\mathbf{3}$ was prepared by means of iterative diastereoselective nucleophilic additions (see $\mathrm{SI}$ ). Macrocycle 4 comprised of 8 total phenylene and cyclohexadiene units was then obtained via dilute SuzukiMiyaura cross-coupling of dibromide 2 with bisboronate 3 . Reductive aromatization of $\mathbf{4}$ with sodium naphthalenide yielded [8]CPP-NB (5). In a similar fashion, Suzuki-Miyaura crosscoupling of bisboronate 6 and dibromide 2 yielded macrocycle 7 comprising 10 (masked) phenylene units. Finally, reductive aromatization of $\mathbf{7}$ yielded [10]CPP-NB (8).

Before polymerization, each monomer was characterized using single crystal X-ray crystallography in addition to UV-vis

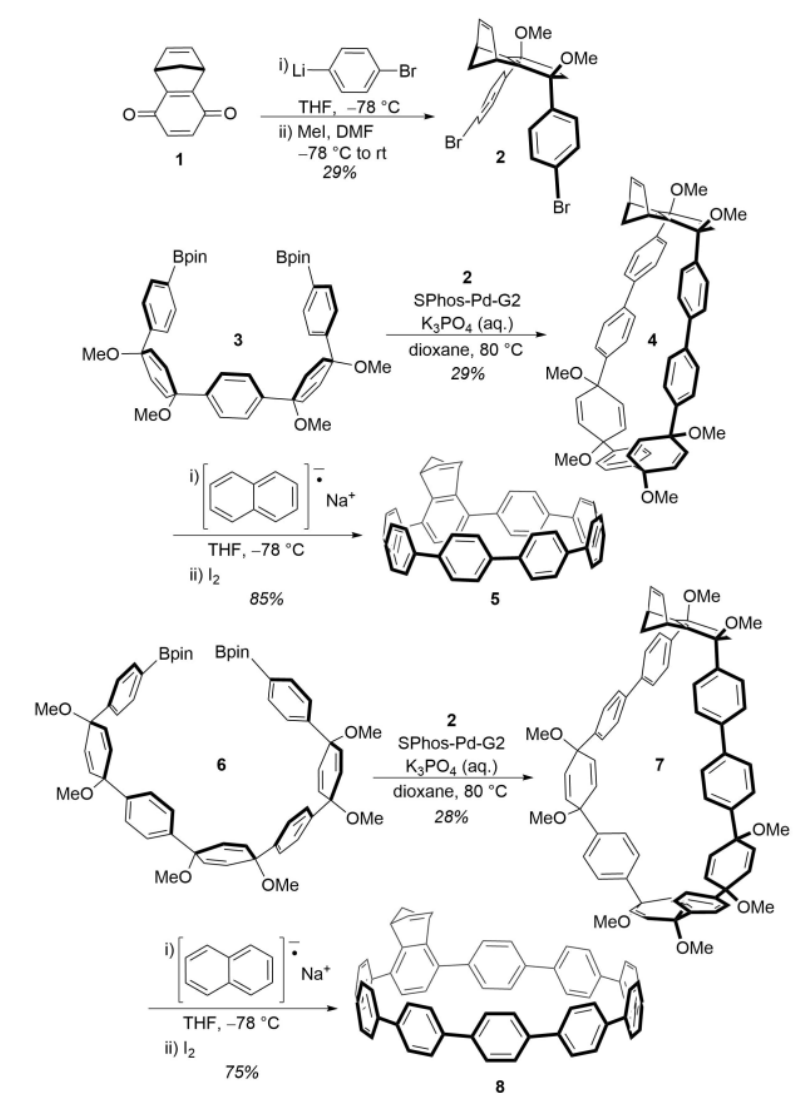

Scheme 1. a) Key steps for synthesizing [8]CPP-NB (5) and [10]CPP-NB (8).

absorbance and fluorescence spectroscopy. X-ray crystallography revealed that [8]CPP-NB crystallized with the norbornene unit disordered over two positions, whereas [10]CPP-NB crystallized with the norbornene alkene exclusively oriented toward the center of the macrocycle. ${ }^{[36]}$ The alkene bond angles in both monomers were between $107^{\circ}$ and $110^{\circ}$ (Fig. S4), typical for norbornenes based on comparison with crystal structures for a variety of norbornene compounds found in the CCDC repository. ${ }^{[37]}$ [8]CPP-NB and [10]CPP-NB exhibit nearly identical characteristics to the corresponding unsubstituted CPPs in terms of both absorbance and emission (Fig, S9; Fig. S10). In underivatized CPPs, both extinction coefficient and quantum yield increase as the number of benzene rings increases, ${ }^{[19,38]}$ and this size-dependent trend holds true for [8]CPP-NB and [10]CPP-NB as well (Fig. S8).

Next, [8]CPP-NB and [10]CPP-NB were each subjected to ROMP using fast-acting bromopyridyl Grubbs G3 initiator ${ }^{[39]}$ in THF (Fig. 2a). These unoptimized conditions successfully produced poly-CPP samples with dispersity values around 1.3 according to gel permeation chromatography (GPC) analysis (Table S1). To our delight, these poly-CPPs were well-soluble in common organic solvents such as chloroform and THF, in stark contrast to linear phenylenes which require solubilizing side chains. ${ }^{1} \mathrm{H}$ NMR spectra of the polymers show broad multiplets characteristic of substituted [8]CPP and [10]CPP (at 7.48 and $7.55 \mathrm{ppm}$, respectively) and extremely broad/flat peaks in the alkyl and alkene regions (see SI), suggesting that the polymers are not stereoregular and likely contain a mix of cis and trans 

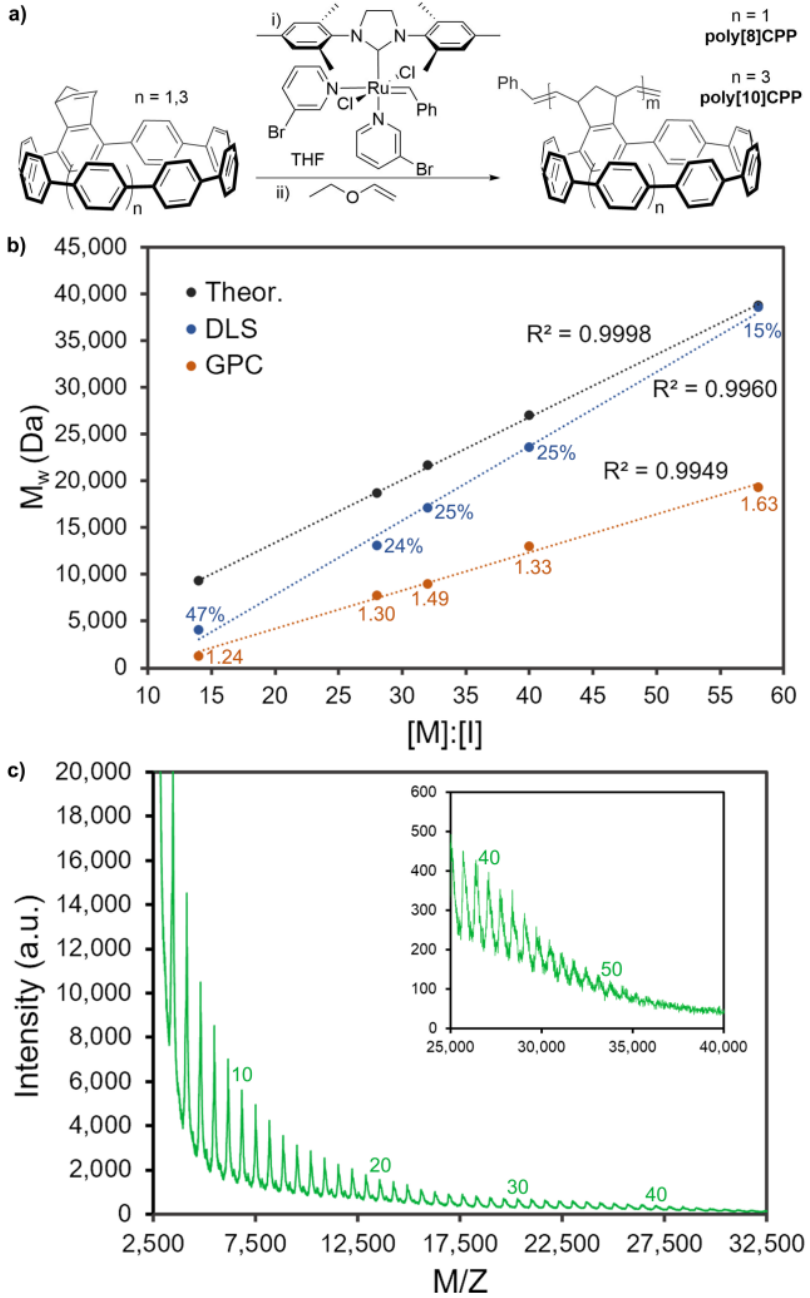

Figure 2. a) Polymerization of CPP-NB monomers under ROMP conditions. b) Poly[8]CPP molecular weight values measured by GPC (orange) and DLS (blue) scale linearly with the molar ratio of monomer to initiator. Dispersity values determined by GPC and DLS are indicated next to the respective data points. c) The MALDI spectrum of a sample of poly[8]CPP shows consistent spacing of $673 \mathrm{Da}$ between peaks. The number of repeat units $(\mathrm{m})$ is labeled periodically above the corresponding peaks. For this sample, with $M_{w} 7,800$ Da measured by GPC and 13,100 Da measured by DLS, MALDI peaks are visible from about 2,500 to $35,000 \mathrm{Da}$.

alkenes. In situ NMR spectroscopy revealed that both monomers are consumed at approximately the same rate (Fig. S5; Fig. S6). To probe the living nature of CPP-NB polymerization, [8]CPP-NB was polymerized over a range of monomer-to-initiator ratios. GPC and dynamic light scattering (DLS) analysis of poly[8]CPP samples revealed a linear trend between monomer-to-initiator ratio and polymer molecular weight (Fig. 2b; Table S1). ${ }^{[0]}$ These results indicate that not only can CPPs be kept intact throughout ROMP, [8]CPP-NB can be polymerized in a living fashion, which opens the door to the construction of more complex polymeric structures containing CPPs. A comparable degree of control over the polymerization of [10]CPP-NB is expected and will be verified in due course. To study the absolute molecular weight distributions of these polymers, several poly-CPP samples were analyzed by matrixassisted laser desorption ionization (MALDI) mass spectrometry (Fig. 2c). The wide range of polymer chain lengths present in the samples precluded quantitative analysis of the spectra. However, the uniform spacing between peaks corresponding with the monomer masses-673 Da for [8]CPP-NB and $825 \mathrm{Da}$ for [10]CPP-NB - further confirmed formation of the desired polymer structures with intact CPP units. Though the upper limits for achievable poly-CPP molecular weights were not tested, MALDI peaks corresponding to polymer chains containing 50 or more repeat units were observed.

To determine if the optical properties of CPPs would be affected by incorporation into polymers, THF solutions of poly[8]CPP and poly[10]CPP were examined using UV-vis absorption and fluorescence spectroscopy. Absorbance and emission spectra of the homopolymers, like those of the monomers, show little change from the spectra of the parent CPPs (Fig, S9; Fig. S10). We then sought to elucidate the tunability of the fluorescence emission of poly-CPPs. At the outset, we postulated that combining [8]CPP-NB and [10]CPPNB units in one polymer would produce an additive emission profile with features from both fluorophores. We suspected that a hybrid polymer with a 1:1 molar ratio of [8]CPP-NB and [10]CPP-NB would emit most intensely around $470 \mathrm{~nm}$ due to [10]CPP-NB being a brighter fluorophore than [8]CPP-NB. To test this, a copolymer was prepared by premixing equimolar amounts of [8]CPP-NB and [10]CPP-NB before addition of initiator to the reaction. Incorporation of both units in the resultant polymer, poly[8]CPP-random-[10]CPP, was confirmed by ${ }^{1} \mathrm{H}$ NMR (Fig. S13). To our surprise, the fluorescence emission of this polymer closely resembled the emission of [8]CPP-NB with a major peak at $529 \mathrm{~nm}$ (Fig. 3). Only a slight shoulder extending from about 415 to $520 \mathrm{~nm}$ revealed any contribution from the [10]CPP-NB units to the overall emission profile. For comparison, we prepared a blend of poly[8]CPP and poly[10]CPP homopolymers in THF. This poly[8]CPP/poly[10]CPP blend displays clear emission contributions from both types of polymers, indicating that the two types of homopolymers are electronically independent in the blend. In contrast, the unexpected emission of copolymerized [8]CPP-NB and [10]CPP-NB suggests that when these hoops are covalently linked in close proximity, interactions emerge that

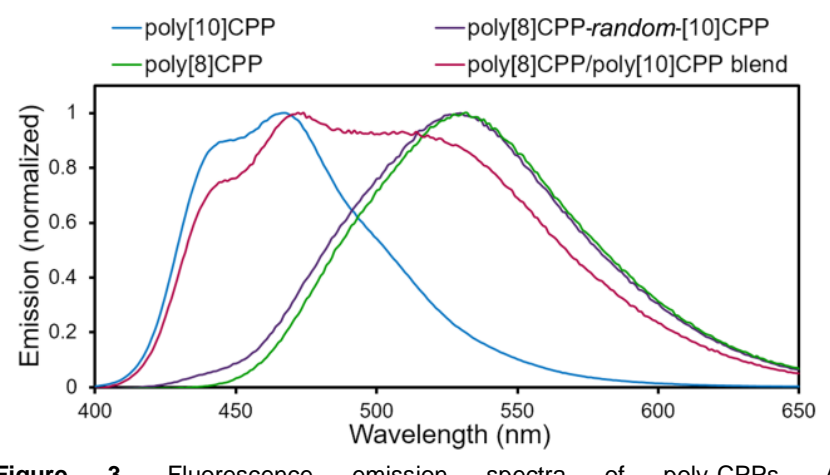

Figure 3. Fluorescence emission spectra of poly-CPPs. A poly[8]CPP/poly[10]CPP blend exhibits characteristics of both poly[8]CPP and poly[10]CPP in its emission profile, whereas the emission of poly[8]CPPrandom-[10]CPP resembles the emission of poly[8]CPP. 
are not observed among individual CPP molecules or blended homopolymers. In poly[8]CPP-random-[10]CPP, the fluorescence of the smaller hoops dominates the overall emission spectrum, indicating energy transfer between the hoop units. A related effect was recently observed in a heterocatenane composed of [9]CPP and [12]CPP. ${ }^{[41]}$ Our observation of energy transfer in CPP copolymers suggests that CPP units can play a role in advanced emissive materials composed of multiple fluorophores.

Finally, we used fluorescence quenching experiments to investigate the host-guest interactions of poly-CPPs with $\mathrm{C}_{60}$, using solutions of the polymers in toluene. Poly[10]CPP exhibits the characteristic fluorescence quenching of [10]CPP by $\mathrm{C}_{60}$ (Fig S11), ${ }^{[23]}$ whereas poly[8]CPP, like [8]CPP, has no inherent affinity for $\mathrm{C}_{60}$ and undergoes only minor dynamic quenching from $\mathrm{C}_{60}$ addition (Fig. S12). Upon $\mathrm{C}_{60}$ addition to the poly[8]CPP/poly[10]CPP blend, the region of the emission spectrum attributed to the contribution from poly[10]CPP, from around $420 \mathrm{~nm}$ to $500 \mathrm{~nm}$, undergoes the greatest quenching effect from $\mathrm{C}_{60}$, while the emission peak at $528 \mathrm{~nm}$ attributed to the emission contribution from poly[8]CPP persists (Fig. 4a). The emission maximum of the blend can be gradually shifted to longer wavelengths by selective quenching of the emission of poly[10]CPP by $\mathrm{C}_{60}$. Aliquots of $\mathrm{C}_{60}$ were then added to $\mathrm{a}$ solution of poly[8]CPP-random-[10]CPP, which resulted in a gradual fluorescence quenching across the entire spectrum (Fig. $4 b)$. Very similar emission and quenching was observed in copolymer samples with $3: 1$ and 1:3 molar ratios of [8]CPP-NB and [10]CPP-NB (Fig. S13). The dramatic difference in quenching between the poly[8]CPP/poly[10]CPP blend and poly[8]CPP-random-[10]CPP reinforces that the close covalent linkage of multiple sizes of CPPs is responsible for these emergent properties.
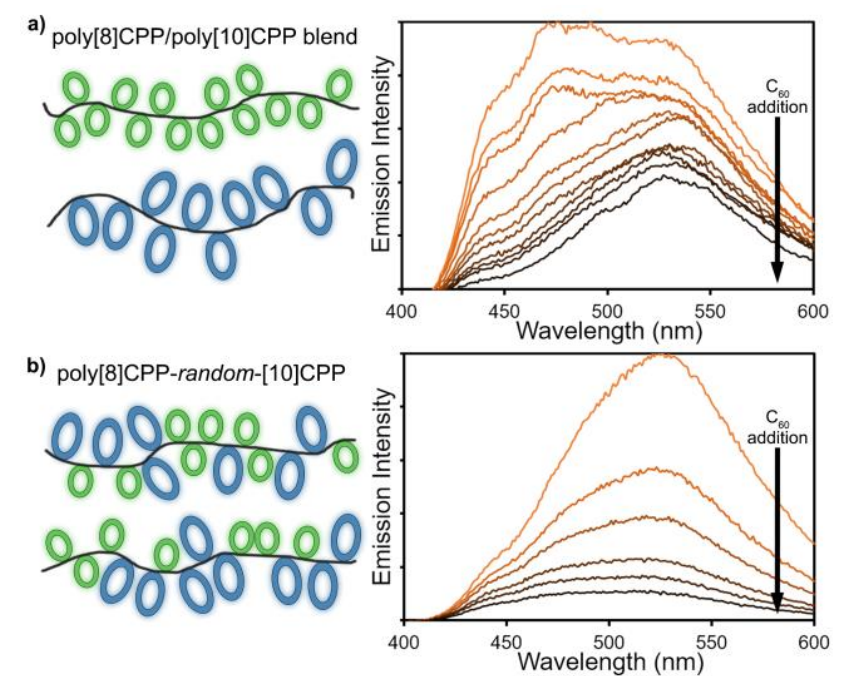

Figure 4. a) A poly[8]CPP/poly[10]CPP blend and b) poly[8]CPP-random[10]CPP, represented pictorially on the left, exhibit drastically different emission profiles and responses to the addition of $\mathrm{C}_{60}$.

In summary, we have introduced CPPs as a new monomer scaffold and demonstrated a straightforward approach to preparing poly-CPPs through ROMP of CPP-NB monomers. Using this approach, the desirable features of CPPs such as solubility, fluorescence, and size-controlled pores are maintained in poly-CPPs. Copolymerizing multiple sizes of CPPNBs provides new avenues to tune the properties of CPP-based polymers. Ultimately, poly-CPPs represent a new form of carbon nanomaterial, uniquely positioned at the intersection of precise organic synthesis and macromolecular chemistry. As the accessible properties in CPP small molecule derivatives multiply, the strategy presented here will allow for any of these properties to be taken advantage of in polymers. In addition, the tools of polymer chemistry can now be used to alter the physical properties and processability of these carbon nanohoop structures and to incorporate them into more complex materials.

\section{Acknowledgements}

This material is based upon work supported by the National Science Foundation Graduate Research Fellowship under Grant No. 1309047 and Department of Energy Grant No. DESC0019017. The authors thank Dr. Casey Check for GPC training, Dr. Nanette Jarenwattananon for help with NMR kinetic studies, Terri Lovell for quantum yield measurements, and Prof. A. J. Boydston for assistance with initial polymerization experiments.

Keywords: cycloparaphenylenes - macrocycles - nanohoops • polymers • ring-opening metathesis polymerization

[1] T. P. Lodge, Macromolecules 2017, 50, 9525-9527.

[2] J. Pyun, X.-Z. Zhou, E. Drockenmuller, C. J. Hawker, J. Mater. Chem. 2003, 13, 2653-2660.

[3] J. F. Lutz, J. M. Lehn, E. W. Meijer, K. Matyjaszewski, Nat. Rev. Mater. 2016, 1, 1-14

[4] C. J. Hawker, K. L. Wooley, Science 2005, 309, 1200-1205.

[5] M. J. Marsella, T. M. Swager, J. Am. Chem. Soc. 1993, 115, 1221412215.

[6] M. Kamachi, X. S. Cheng, T. Kida, A. Kajiwara, M. Shibasaka, S. Nagata, Macromolecules 1987, 20, 2665-2669.

[7] P. Lo Meo, G. Lazzara, L. Liotta, S. Riela, R. Noto, Polym. Chem. 2014, 5, 4499-4510.

[8] X. Ji, R.-T. Wu, L. Long, C. Guo, N. M. Khashab, F. Huang, J. L. Sessler, J. Am. Chem. Soc. 2018, 140, 2777-2780.

[9] S. M. Barbon, M. Rolland, A. Anastasaki, N. P. Truong, M. W. Schulze, C. M. Bates, C. J. Hawker, Macromolecules 2018, 51, 6901-6910.

[10] A. Alsbaiee, B. J. Smith, L. Xiao, Y. Ling, D. E. Helbling, W. R. Dichtel, Nature 2015, 529, 190-194.

[11] L. P. Skala, A. Yang, M. J. Klemes, L. Xiao, W. R. Dichtel, J. Am. Chem. Soc. 2019, jacs.9b06749. 
[12] S. Chaudhuri, M. Mohanan, A. V Willems, J. A. Bertke, N. Gavvalapalli, Chem. Sci. 2019, 10, 5976-5982.

[13] T. Murakami, B. V. K. J. Schmidt, H. R. Brown, C. J. Hawker, Macromolecules 2015, 48, 7774-7781.

[14] E. A. Appel, J. del Barrio, X. J. Loh, O. A. Scherman, Chem. Soc. Rev. 2012, 41, 6195-6214.

[15] B. V. K. J. Schmidt, C. Barner-Kowollik, Angew. Chem. Int. Ed. 2017, 56, 8350-8369; Angew. Chem. 2017, 129, 8468-8488.

[16] Q. Wu, P. M. Rauscher, X. Lang, R. J. Wojtecki, J. J. de Pablo, M. J. A. Hore, S. J. Rowan, Science 2017, 358, 1434-1439.

[17] Z. Niu, H. W. Gibson, Chem. Rev. 2009, 109, 6024-6046.

[18] K. Ito, Polym. J. 2007, 39, 489-499.

[19] E. R. Darzi, R. Jasti, Chem. Soc. Rev. 2015, 44, 6401-6410.

[20] E. R. Darzi, E. S. Hirst, C. D. Weber, L. N. Zakharov, M. C. Lonergan, R. Jasti, ACS Cent. Sci. 2015, 1, 335-342.

[21] T. C. Lovell, C. E. Colwell, L. N. Zakharov, R. Jasti, Chem. Sci. 2019, 10, 3786-3790.

[22] Y. Xu, M. Von Delius, Angew. Chem. Int. Ed. 2019, Accepted Article; Angew. Chem. 2019, Accepted Article.

[23] T. Iwamoto, Y. Watanabe, T. Sadahiro, T. Haino, S. Yamago, Angew. Chem. Int. Ed. 2011, 50, 8342-8344; Angew. Chem. 2011, 123, 8492-8494.

[24] J. Xia, J. W. Bacon, R. Jasti, Chem. Sci. 2012, 3, 3018-3021.

[25] J. M. Van Raden, B. M. White, L. N. Zakharov, R. Jasti, Angew. Chem. Int. Ed. 2019, 58, 7341-7345; Angew. Chem. 2019, 131, 7419-7423.

[26] B. M. White, Y. Zhao, T. E. Kawashima, B. P. Branchaud, M. D. Pluth, R. Jasti, ACS Cent. Sci. 2018, 4, 1173-1178.

[27] H. Omachi, T. Nakayama, E. Takahashi, Y. Segawa, K. Itami, Nat. Chem. 2013, 5, 572-576.
[28] B. D. Steinberg, L. T. Scott, Angew. Chem. Int. Ed. 2009, 48, 54005402; Angew. Chem. 2009, 121, 5504-5507.

[29] R. Jasti, C. R. Bertozzi, Chem. Phys. Lett. 2010, 494, 1-7.

[30] T. J. Sisto, L. N. Zakharov, B. M. White, R. Jasti, Chem. Sci. 2016, 7, 3681-3688.

[31] E. Kayahara, R. Qu, S. Yamago, Angew. Chem. Int. Ed. 2017, 56, 10428-10432; Angew. Chem. 2017, 129, 10564-10568.

[32] C. Slugovc, Macromol. Rapid Commun. 2004, 25, 1283-1297.

[33] A. Leitgeb, J. Wappel, C. Slugovc, Polymer 2010, 51, 2927-2946.

[34] R. Jasti, J. Bhattacharjee, J. B. Neaton, C. R. Bertozzi, J. Am. Chem Soc. 2008, 130, 17646-17647.

[35] The relatively low yield for this step (29\%) reflects the fact that 2 was isolated by precipitation from a mixture of diastereomers. The stereochemistry of $\mathbf{2}$ was confirmed by x-ray crystallography (Fig. S1).

[36] CCDC 1949617, 1949616, and 1949615 contain the supplementary crystallographic data for this paper. These data can be obtained free of charge from The Cambridge Crystallographic Data Centre.

[37] "The Cambridge Crystallographic Data Centre," can be found under https://www.ccdc.cam.ac.uk/.

[38] E. R. Darzi, T. J. Sisto, R. Jasti, J. Org. Chem. 2012, 77, 6624-6628.

[39] T.-L. Choi, R. H. Grubbs, Angew. Chem. Int. Ed. 2003, 42, 17431746; Angew. Chem. 2003, 115, 1785-1788.

[40] GPC analysis was conducted using polystyrene (PS) standards, and the discrepancies between theoretical and measured molecular weights for poly-CPPs reflect the significant differences in hydrodynamic behavior between poly-CPPs and PS.

[41] Y. Segawa, M. Kuwayama, Y. Hijikata, M. Fushimi, T. Nishihara, J. Pirillo, J. Shirasaki, N. Kubota, K. Itami, Science 2019, 365, 272276. 
Entry for the Table of Contents

\section{COMMUNICATION}

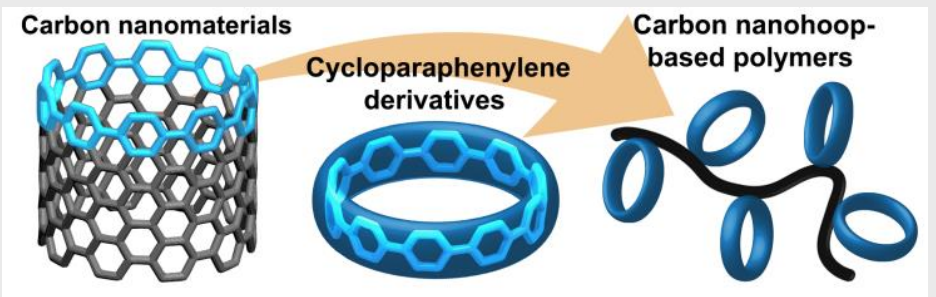

Cyclic building blocks: The first polymers constructed from cycloparaphenylenes (CPPs), a family of strained, pi-rich macrocycles, are reported. The distinctive fluorescence and host-guest chemistry of the monomers are preserved in polyCPPs, and emergent properties are observed.
Ruth L. Maust, Penghao Li, Lev N.

Zakharov, Ramesh Jasti*

Page No. - Page No.

Cycloparaphenylene-Norbornene Monomers Afford Access to Carbon Nanohoop-Based Polymers 